\title{
New Insights into the Cell Biology of Hematopoietic Progenitors by Studying Prominin-1 (CD133)
}

\author{
Nicola Bauer ${ }^{\mathrm{a}}$ Ana-Violeta Fonseca ${ }^{\mathrm{a}}$ Mareike Florek $^{\mathrm{b}}$ Daniel Freund $^{\mathrm{a}}$

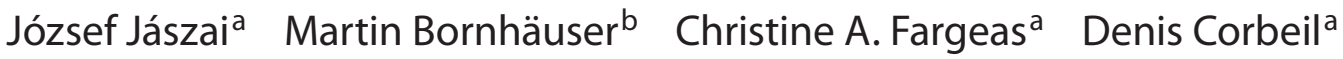 \\ aTissue Engineering Laboratories, Biotec, Technische Universität Dresden, and ${ }^{b}$ Medical Clinic and Polyclinic I, \\ University Hospital Carl Gustav Carus, Dresden, Germany
}

\section{Key Words}

Hematopoietic stem cells • Polarity • Plasma membrane • Cell migration $\cdot$ Cell division

\begin{abstract}
Prominin-1 (alias CD133) has received considerable interest because of its expression by several stem and progenitor cells originating from various sources, including the neural and hematopoietic systems. As a cell surface marker, prominin-1 is now used for somatic stem cell isolation. Its expression in cancer stem cells has broadened its clinical value, as it might be useful to outline new prospects for more effective cancer therapies by targeting tumor-initiating cells. Cell biological studies of this molecule have demonstrated that it is specifically concentrated in various membrane structures that protrude from the planar areas of the plasmalemma. Prominin-1 binds to the plasma membrane cholesterol and is associated with a particular membrane microdomain in a cholesterol-dependent manner. Although its physiological function is not yet determined, it is becoming clear that this cell surface protein, as a unique marker of both plasma membrane protrusions and membrane microdomains, might reveal new aspects of the cell biology of rare stem and cancer stem cells. The aim of this review is to outline the recent discoveries regarding the dynamic reorganization of the plasma membrane of rare CD133+ hematopoietic progenitor cells during cell migration and division.
\end{abstract}

Copyright $\odot 2007$ S. Karger AG, Basel

\section{KARGER}

Fax +4161306 1234

E-Mail karger@karger.ch

www.karger.com
(C) 2007 S. Karger AG, Basel

$1422-6405 / 08 / 1882-0127 \$ 24.50 / 0$

Accessible online at:

www.karger.com/cto

\section{Introduction}

The cell biology of stem and progenitor cells is now an emerging field that will allow us to better understand and eventually control the essential cellular mechanisms underlying the unique properties of these cells such as, for instance, their self-renewal capacity and their ability to differentiate into specialized mature cells. It appears that the cellular environment in the vicinity of these stem cells plays a crucial role in the maintenance of their stem cell properties. Thus, the migration and/or interaction of stem and progenitor cells with a particular cellular niche are of central importance for their cell biology. Nevertheless, the molecular and cellular bases of these events remain poorly understood, although they are certainly of potential therapeutic relevance. This lack of knowledge is mainly due to the fact that the stem and progenitor cells are rare and/or hidden in a large heterogeneous cell pop-
Abbreviations used in this paper
CXCR 4 C-X-C chemokine receptor 4
HSPCs hematopoietic stem and progenitor cells
ICAM intercellular adhesion molecule
MSCs mesenchymal stromal cells
PSGL P-selectin glycoprotein ligand
SDF-1 stromal cell-derived factor-1 
ulation. The discovery of new cell surface markers, which allow their prospective isolation and characterization, opens a new avenue to study the stem cell biology. In this review, we will focus on one of the most extensively studied markers named prominin-1 (alias CD133). We will put special emphasis on the stem and progenitor cells found in the hematopoietic system.

\section{Prominin-1 (CD133)}

\section{A Stem Cell Marker}

Since its discovery 10 years ago, prominin-1 has received considerable interest given its expression by several somatic stem and progenitor cells originating from various sources, including the neural and hematopoietic systems, and in embryonic stem cell-derived progenitors [Weigmann et al., 1997; Yin et al., 1997; Kania et al., 2005; for review, see Fargeas et al., 2006]. Murine prominin-1 was identified as a novel marker of neuroepithelial cells, primary progenitor cells of the mammalian central nervous system [Weigmann et al., 1997], whereas its human counterpart constituted a new hematopoietic stem and progenitor cell (HSPC) marker (initially referred to as AC133 antigen) [Miraglia et al., 1997; Yin et al., 1997]. As a cell surface marker, prominin-1 is now used for somatic stem cell isolation [Yin et al., 1997; Uchida et al., 2000; Richardson et al., 2004; Lee et al., 2005]. CD133+ stem and progenitor cells might become clinically important, particularly with regard to brain injury/disease and bone marrow transplantation (see below). Prominin-1 as such might open new perspectives of gene therapy in stem cell tissue engineering [Fargeas et al., 2006; Brenner et al., 2007; Jászai et al., 2007a].

It is important to note that, although various stem and progenitor cells express prominin-1, its expression is not limited to primitive cells. For instance, prominin-1 is detected in several epithelia in adult mice and humans where it appears to be restricted to the apical (luminal) side [Weigmann et al., 1997; Florek et al., 2005; Lardon et al., 2007; Jászai et al., 2007b; for reviews concerning its expression pattern, see Corbeil et al., 2001; Fargeas et al., 2006].

\section{Prominin-1 and Plasma Membrane Protrusions}

Prominin-1 is a pentaspan membrane glycoprotein that exhibits a profound preference for membrane curvature, which is illustrated by its concentration in various structures having in common that they protrude from the planar areas of the plasmalemma such as microvilli, cilia and at the leading edge of lamellipodia [Weigmann et al., 1997; Corbeil et al., 2000; for review, see Corbeil et al., 2001]. The concentration of prominin-1 in 2 distinct types of plasma membrane protrusions with different structural bases (actin for the microvillus and tubulin for the cilium) suggests that the mechanism underlying its remarkable subcellular localization is independent of a direct interaction with the actin or tubulin network, unless prominin-1 binds directly to both types of cytoskeletal elements [Dubreuil et al., 2007; Florek et al., 2007]. The latter scenario is highly unlikely, since the deletion of its C-terminal cytoplasmic tail (the only plausible cytoskeleton-interacting domain) does not prevent its concentration in plasma membrane protrusions [Corbeil et al., 1999].

Several splice variants affecting the protein sequence of prominin-1 have been identified [Yu et al., 2002; Fargeas et al., 2003, 2004, 2007]. Although they display a broad range of expression, all appear to be confined to plasma membrane protrusions. For instance, a specific prominin-1 variant named s3 [for nomenclature, see Fargeas et al., 2007] is found in the myelin sheath [Corbeil et al., manuscript under revision], which represents a specialized plasma membrane protrusion of oligodendrocytes. Another variant, s6, is located in the tail of developing spermatozoa [Fargeas et al., 2004]. Prominin-1 is also concentrated in plasma membrane evaginations located at the base of the outer segment of photoreceptor cells, which are the precursor structures in the biogenesis of photoreceptor disks [Maw et al., 2000]. This particular subcellular localization is interesting, given that mutations in the human PROMININ-1 gene are associated with a retinal degeneration suggesting that this membrane protein might play a role in disk morphogenesis [Maw et al., 2000; Jászai et al., 2007a; Zhang et al., 2007].

\section{Prominin-1 and Membrane Microdomains}

Prominin-1 is a cholesterol-binding glycoprotein which is associated with a membrane microdomain (often referred to as 'lipid microdomain' or 'lipid raft') [for a unified definition, see Pike, 2006] containing the ganglioside GM1 in a cholesterol-dependent manner [Röper et al., 2000; Janich and Corbeil, 2007]. Membrane microdomains are viewed as liquid-ordered domains that are more tightly packed than the surrounding phase of the bilayer. They are enriched in sterol and sphingolipids present in the exoplasmic membrane leaflet and contain a specific set of membrane and peripheral proteins [Pike, 2004]. Interestingly, the integrity of these membrane mi- 
crodomains appears essential to the maintenance of prominin-1 localization in plasma membrane protrusions as demonstrated by its dispersion upon cholesterol depletion [Röper et al., 2000].

The association of prominin-1 with membrane microdomains, which are known to be implicated in several signaling cascades by allowing the formation of active transduction complexes [Simons and Toomre, 2000], is highly interesting particularly with regard to its expression in stem and progenitor cells. The prominin-1-containing membrane microdomains might carry all molecular determinants necessary to maintain the stem cell properties and their loss, for example via an asymmetric cell division, might contribute to cell differentiation (see below) [Kosodo et al., 2004; Fargeas et al., 2006].

\section{Prominosomes}

Although tightly associated with the plasma membrane via its 5 transmembrane segments, prominin- 1 is nonetheless released into various physiological body fluids including saliva, urine, seminal fluid and tears [Marzesco et al., 2005; Florek et al., 2007]. In these fluids, prominin-1 is associated with small membrane vesicles $(50-80 \mathrm{~nm})$, referred to as prominosomes [Marzesco et al., 2005], which might arise, at least in part, from microvilli and/or primary cilia where prominin-1 appears concentrated at their tips [Weigmann et al., 1997; Dubreuil et al., 2007; Florek et al., 2007]. In the developing central nervous system of mammals, prominin-1 is not only associated with small membrane vesicles, but also with apical midbodies that are released into the neural tube fluid [Dubreuil et al., 2007]. Their role is currently unknown. Nevertheless, we can hypothesize that the release of these various membrane particles by stem and progenitor cells, for example neuroepithelial [Marzesco et al., 2005; Dubreuil et al., 2007] and hematopoietic progenitors [Bauer et al., manuscript in preparation], might be a means of disposal of a 'stem cell-specific membrane microdomain', allowing these cells to modify their stem and progenitor cell properties (see above). Alternatively, the prominin-1containing membrane particles might play a role in intercellular communication by carrying specific signaling molecules. Such a scenario, without excluding the former one, would be particularly relevant in the context of tissue formation.

Although the physiological function of prominin-1 is not yet determined, it becomes clear that this cholesterolbinding protein, as a unique marker of both plasma membrane protrusions and membrane microdomains, might reveal new aspects of the cell biology of stem and pro- genitor cells, particularly with regard to their plasma membrane organization during cell migration and division.

\section{CD133+ HSPCs}

\section{Clinical Value}

Hematopoiesis is maintained by a small number of HSPCs, which are able to proliferate and differentiate into all lineages of the hematopoietic system. Since these rare primitive cells reside within a large heterogeneous cell population, their proper isolation and in vivo characterization inside the bone marrow compartment rely on the presence and/or absence of specific plasma membrane markers. Throughout the last decade, monoclonal antibodies against the human sialomucin CD34 [Krause et al., 1996] have been used to isolate HSPCs. These cells were initially selected using immunoadsorptive methods and fluorescence-activated cell sorting, but soon the selection of CD34+ HSPCs by paramagnetic bead-coupled antibodies became the method of choice in clinical stem cell transplantation [Beaujean, 1997].

Prominin-1 is expressed on a subpopulation of CD34+ HSPCs derived from various sources including fetal liver and bone marrow, adult bone marrow, cord blood and mobilized peripheral blood [Miraglia et al., 1997; Yin et al., 1997; de Wynter et al., 1998; Corbeil et al., 2000]. The proportion of CD133+ cells within the CD34+ population varies depending on the source of HSPCs (35-90\%) with large discrepancies in the data reported by independent research groups [Matsumoto et al., 2000]. Moreover, a unique population of CD34- HSPCs expressing prominin-1 with primitive stem cell properties has been reported [Gallacher et al., 2000; Kuci et al., 2003]. The CD133+ HSPCs have the capacity to reconstitute the entire immune system of lethally irradiated mice [de Wynter et al., 1998]. Besides their hematopoietic capacity, these cells are capable of in vitro differentiation into neuronal cells [Padovan et al., 2003], endothelial cells [Gehling et al., 2000; Quirici et al., 2001] and myoblasts [Torrente et al., 2004], suggesting broader multipotential capacities of CD133+ populations compared to the CD34+ ones.

Interestingly, an immunomagnetic selection of CD133+ HSPCs allowed the enrichment of a sufficient amount of cells to perform hematopoietic stem cell transplantation [Gordon et al., 2003], and pilot trials with leukemic children have proven the feasibility of CD133+ selection for allogeneic transplantation [Koehl et al., 2002; 
Lang et al., 2004]. Similarly, we could demonstrate a rapid establishment of donor dendritic cell chimerism after allogeneic transplantation of $\mathrm{CD} 133+$ selected cells [Bornhäuser et al., 2005]. Other studies have shown the successful transplantation of haploidentically mismatched peripheral blood stem cells using CD133+ purified stem cells [Bitan et al., 2005]. Thus, the immunomagnetic isolation procedure of HSPCs based on prominin-1 appears to be an interesting alternative to CD34 [Freund et al., 2006a].

Prominin-1, in addition to being normally expressed on the surface of HSPCs, is detected in various malignant hematopoietic diseases including acute and chronic myeloid as well as lymphoblastic leukemias [for review, see Bhatia, 2001]. In most leukemias, both prominin-1 and CD34 are expressed, but some investigators have identified leukemic blasts harboring prominin-1 but not CD34 [Kratz-Albers et al., 1998]. The dual expression of prominin-1 by HSPCs and by their leukemic counterparts is highly consistent with the cancer stem cell hypothesis, which suggests that not all cells in a tumor have the same capacity to proliferate [Bonnet and Dick, 1997]. In fact, the CD133+ cells might represent a unique population of cancer stem cells that possess the ability to proliferate and maintain their self-renewal capacity extensively in an uncontrolled fashion. A similar conclusion has been drawn recently based on the expression of prominin-1 in solid cancer cells that are capable of initiating tumor growth [Singh et al., 2004; O'Brien et al., 2007; Ricci-Vitiani et al., 2007]. Thus, prominin-1 might be useful to outline new prospects for more effective cancer therapies by targeting tumor-initiating cells.

\section{Cell Shape and Migration}

The trafficking of HSPCs is a fundamental process not only during ontogenesis where the primitive HSPCs move by sequential events from the yolk sac and/or aorta-gonad-mesonephros to the fetal liver/spleen and finally to the adult bone marrow, but also during steady-state homeostasis - a phenomenon occurring throughout the entire adult life. In many aspects, the homing of infused HSPCs to the adult bone marrow microenvironment and their mobilization from this specialized endosteal/endothelial niche [for reviews, see Adams and Scadden, 2006; Kiel and Morrison, 2006; Li and Li, 2006; Wilson and Trumpp, 2006] to the peripheral blood, following administration of chemotherapeutic agents, mimic the physiological migration of HSPCs during embryogenesis. The orchestrated processes underlying the HSPC trafficking require a high degree of molecular specificity [Hardy,
1995; Papayannopoulou et al., 1995; Cancelas et al., 2006] and possibly plasma membrane flexibility. Despite the central role of these events in hematopoietic stem cell biology and their therapeutic relevance, their molecular and cellular bases still remain poorly understood [Wright et al., 2001].

It is likely that the HSPC trafficking to some extent mimics the multistep migration process of peripheral leukocytes to inflammatory sites [Butcher and Picker, 1996; Luster et al., 2005]. An important prerequisite for such migration is the acquisition of a polarized morphology through reorganization of the cytoskeleton elements that is conducted, at least in part, by the activation of small Rho GTPase proteins [for review, see Sanchez-Madrid and del Pozo, 1999] and results in the formation of a lamellipodium-like structure at the front side (leading edge) and a uropod at the rear pole. The uropod is a specialized pseudopod-like projection with important functions including motility and recruitment of bystander cells.

Using a coculture consisting of human CD133/34+ HSPCs growing on a monolayer of human primary multipotent mesenchymal stromal cells (MSCs) in the presence of relevant cytokines [for technical details, see Freund et al., 2006b], we and others have shown that migrating HSPCs exhibit a leukocyte-like polarized morphology (fig. 1a) [Fruehauf et al., 2002; Giebel et al., 2004; Freund et al., 2006b; Wagner et al., 2007]. Interestingly, several membrane proteins are selectively segregated upon polarization. For instance, prominin-1 is selectively concentrated in the uropod (fig. 1b), whereas the $\mathrm{G}$ protein-coupled 7-span transmembrane receptor CXCR4 - a chemokine stromal cell-derived factor 1 (SDF-1) receptor - is found at the leading edge [Giebel et al., 2004] in agreement with its role in directional cell migration through an SDF-1 gradient [for reviews, see Lapidot and Kollet, 2002; Dar et al., 2006]. Numerous adhesion molecules, including CD43, CD44, intercellular adhesion molecule-1/3 and P-selectin glycoprotein ligand-1, are enriched in the uropod as shown for prominin-1 (fig. 1b, inset). Other molecules such as CD34 and CD45 are randomly distributed [Giebel et al., 2004; Fonseca and Corbeil, unpubl. data].

Given that the selective enrichment of prominin-1 in plasma membrane protrusions depends on a particular cholesterol-based membrane microdomain [Röper et al., 2000], this observation points out that the lipid composition of the uropod might be distinct. Indeed, 2 raft-associated gangliosides, $\mathrm{GM}_{1}$ (as revealed by the binding of the cholera toxin $\mathrm{B}$ subunit) and $\mathrm{GM}_{3}$, are segregated in 


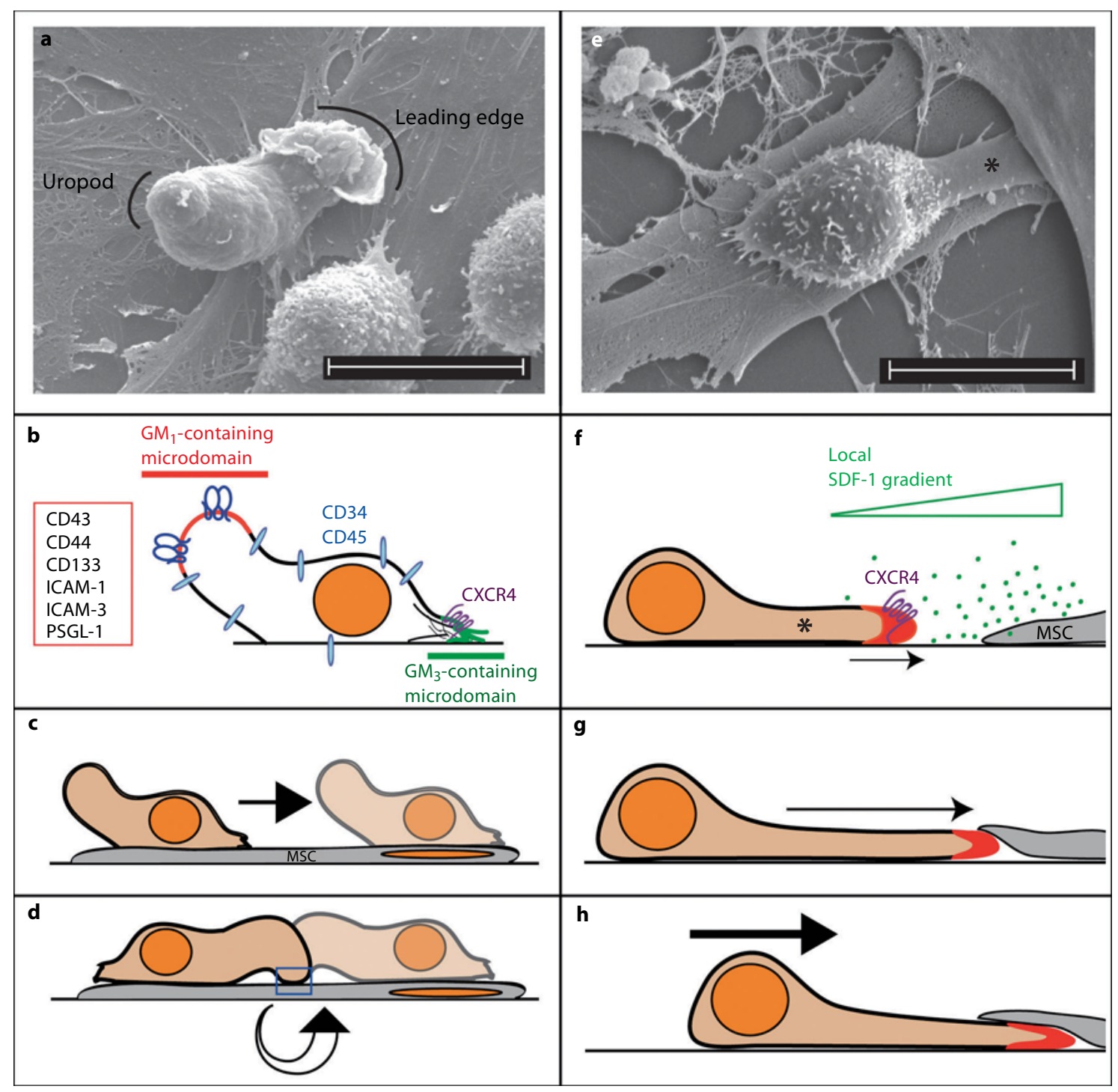

Fig. 1. HSPCs exhibit 2 distinct modes of locomotion. a, e CD34+ MACS-immunoisolated HSPCs from leukapheresis products were cultured for 7 days on primary human MSCs prior to analysis by scanning electron microscopy. a-d The first type of locomotion involves the formation of a uropod at the rear pole and a leading edge at the front pole (a; solid lines). Both types of plasma membrane protrusions contain a specific membrane microdomain - the uropod is enriched in $\mathrm{GM}_{1}$ ganglioside, whereas the leading edge is enriched in $\mathrm{GM}_{3}$ ganglioside (b; red and green, respectively). In addition to prominin-1 (CD133), several cell adhesion molecules are concentrated in $\mathrm{GM}_{1}$-based microdomain (b; red box). CXCR4 is found at the leading edge and others such as CD34 and CD45 are distributed in a nonpolarized fashion (b). This mode of locomotion - where the uropod at the rear pole is up - is similar to that used by migrating leukocytes (c; arrow indicates the direction of migration). When the uropod at the rear pole gets in contact with the MSC, the HSPCs stop their migration and start to rotate around the axis generated by the contact point (d; curved arrow). The blue box highlights the contact zone between HSPC and MSC, which might be important for cellular communication. e-h The second type of locomotion involves the formation of a large magnupodium emerging from an HSPC (e, f; asterisks) that will grow and move underneath an MSC (g; arrow). When the magnupodium senses the appropriate niche, the entire cell body of the HSPC will move therein (h; arrow). Please note that during these movements the HSPC does not exhibit any uropod structure, which stands in contrast to those with a highly motile locomotion (a-d). The presence of prominin-1 at the edge of the magnupodium suggests that a specific membrane microdomain (red) might endow this plasma membrane subdomain with appropriate receptor/adhesion molecules such as CXCR4 (f). SDF-1 (green dots) secreted by MSCs might attract and guide the magnupodium via its receptor CXCR4 (f). The original picture in e was previously presented in Freund et al. [2006b]. ICAM = Intercellular adhesion molecule; $\mathrm{PSGL}=\mathrm{P}$-selectin glycoprotein ligand; $\mathrm{SDF}=$ stromal cell-derived factor. Scale bars $=10 \mu \mathrm{m}$. 
migrating HSPCs (fig. $1 \mathrm{~b}$ ) $-\mathrm{GM}_{1}$ is enriched in the uropod [Gillette et al., 2006], whereas $\mathrm{GM}_{3}$ is found in the leading edge (fig. 1b) [Giebel et al., 2004]. A similar observation has previously been made with migrating leukocytes, thus reinforcing the parallel in the polarization process between these 2 cell types [Gómez-Moutón et al., 2001]. The concentration of prominin-1 in $\mathrm{GM}_{1}$-rich, but not $\mathrm{GM}_{3}$-rich membrane microdomains appears to be a general phenomenon, since a similar event occurs in polarized epithelial cells [Janich and Corbeil, 2007]. Therefore, the polarization of migrating HSPCs might be driven not only by cytoskeleton remodeling directed, for instance, by small Rho GTPase [Giebel et al., 2004; Konakahara et al., 2004; Cancelas et al., 2006; Ghiaur et al., 2006; Yang et al., 2007], but also by a reorganization of their plasma membrane in terms of lipid microdomains. The latter might be required to concentrate and/or retain a particular set of membrane proteins in a given subdomain, such as uropod or leading edge. It is interesting to note that several adhesion membrane proteins are found in the uropod (fig. 1b) and, as a consequence, when this trailing edge protrusion touches the feeder cells, the HSPCs stop their migration (fig. 1c) and start to rotate around the axis generated by the contact point (fig. 1d) [Wagner et al., 2005; Fonseca and Corbeil, unpubl. data].

We have recently demonstrated that CD133+ HSPCs adopt various other morphologies than the leukocytelike migrating structure. They develop several types of plasma membrane protrusions, including microvillus, magnupodium and lamellipodium, where prominin- 1 is selectively concentrated [Corbeil et al., 2000; Freund et al., 2006b]. Although little is known about the physiological role of these protrusions, they are generally considered to be involved in the homing of HSPCs into the bone marrow [Frimberger et al., 2001]. For instance, the large magnupodium moving underneath MSCs might act as a searching-sensor process to guide HSPCs to an appropriate microenvironment (fig. le-h) [Konakahara et al., 2004; Wagner et al., 2005; Freund et al., 2006b]. The observation that similar plasma membrane protrusions (named proteopodia), which are themselves highly motile even when the cells are not in rapid motion, were induced by the chemotactic factor SDF-1 is in agreement with such a function [Frimberger et al., 2001]. It seems highly unlikely that the concentration of the CXCR4 receptor at the edge of the magnupodium [Freund et al., 2006b] and the secretion of SDF-1 by MSCs would be unrelated events (fig. 1f) [Freund et al., 2006b; van Overstraeten-Schlogel et al., 2006]. The concentration of prominin-1, contrary to CD34, at the edge of the magnupodium suggests that a specific membrane microdomain might indirectly trigger the formation of such a searching-sensor process by clustering CXCR4 and other appropriate adhesion molecules, such as CD54 and CD29 [Konakahara et al., 2004; Freund et al., 2006b]. The cytoplasmic machinery, notably the Rho GTPase/LIM kinase $1 /$ cofilin pathway through CD29, might also be involved in their biogenesis, as previously suggested [Konakahara et al., 2004]. It remains to be determined if a particular ganglioside is found therein, but it seems that an active remodeling of the plasma membrane is required.

Thus HSPCs display 2 distinct modes of locomotion, which result in important morphological changes (fig. 1). The first mimics the migration of highly motile leukocytes with the formation of a uropod subdomain at the rear pole (fig. 1a-d). This type of locomotion might play a role in the trafficking of HSPCs inside the blood vessel and during the transendothelial migration. The second involves the generation of a searching-sensor magnupodium at the front side that might guide HSPCs to the final destination inside the bone marrow medullary cavity (fig. le-h). Further studies involving, for instance, an intravital microscopy model are needed to establish the physiological relevance of these migration behaviors. Our increasing knowledge concerning the migration mechanisms of HSPCs should help to improve the process of homing and engraftment of infused cells in medical application.

\section{Intercellular Communication}

An important aspect of the cellular biology of HSPCs is to understand how these cells can communicate with their cellular microenvironment known as the stem cell niche. Various mechanisms for the exchange of molecular information between cells have been documented. The secretion of molecules and their interaction with their receptors located on the target cells or contact-dependent signaling are good examples. The SDF-1 and CXCR4 signaling axis falls into the first category [Faber et al., 2007], whereas the Notch/Delta signaling falls into the second [Varnum-Finney et al., 2000]. Gerdes et al. [2007] have proposed a third way for mammalian cells to communicate based on the formation of thin membrane channels between them. Remarkably, we could observe that HSPCs develop peculiar thin plasma membrane structures (diameter $50-80 \mathrm{~nm}$ ), which link 2 or more adjacent HSPCs, leading to the formation of complex cellular networks [Freund et al., 2006b]. These structures 
are highly reminiscent of the tunneling nanotubes that allow the direct intercellular transfer of diverse components (molecules, small and large organelles such as mitochondria) and might act, in contrast to gap junctions, at a long range [Rustom et al., 2004]. It remains to be established which information HSPCs might share, but it is tempting to speculate that such membrane processes might allow them to sense the presence or absence of their neighbors within the niche. As a consequence, 2 scenarios are possible: first, a signal might be transduced within the connected cells, which will lead to a steady state, that is, nonproliferation. Second, the absence of interaction might result in proliferation, permitting the replenishment of the niche.

Lippincott-Schwartz and colleagues have recently proposed an alternative way for the HSPCs to communicate with their cellular environment, which involves the contact zone between the HSPCs' uropod and the feeder cells (fig. 1d, blue box) [Gillette et al., 2006]. This cell-cell contact zone might serve as an intimate room for intercellular communication where HSPCs' plasma membrane proteins including prominin-1 are transferred to the feeder cells [Gillette et al., 2006]. Although such an event would have an important implication for the interaction/ communication of HSPCs with their cellular niche, the cellular mechanism underlying this transfer of molecules is currently unknown. However, knowing that prominin1 is associated with small membrane vesicles that are released not only from the neuroepithelial cells (see above), but also from HSPCs [Bauer et al., manuscript in preparation], it is tempting to speculate that these vesicles are the carrier involved in such molecule shuttling. It cannot be a coincidence that prominin-1 is concentrated in the uropod and that this particular plasma membrane subdomain is implicated in the budding of human immunodeficiency virus type 1 particles produced by infected $\mathrm{T}$ cell lines [Nguyen and Hildreth, 2000]. However, we cannot exclude other sources for these particles, since prominin-1 also appears to be associated with multivesicular bodies in HSPCs [Bauer et al., 2007, manuscript in preparation] and it might be released into the extracellular environment associated with exosomes [Février and Raposo, 2004].

Clearly, the cellular communication of HSPCs is a novel research field that requires further investigation, and the development of ex vivo cultures [Dexter et al., 1977; Majumdar et al., 2000; Kadereit et al., 2002; Wagner et al., 2005; Freund et al., 2006b], mimicking the bone marrow niche, might help to address this issue.

Prominin-1 and the Hematopoietic System

\section{Cellular Polarization and Cell Division}

Little is known about the plasma membrane polarity of HSPCs and the distribution of membrane components during cell division. The identification of intrinsic factors playing a role in the maintenance of their stem cell properties is highly important. The symmetric versus asymmetric distribution of such determinants during cell division might govern the fate of the daughter cells. Consequently, new cell-based therapeutic strategies might eventually arise from the manipulation of these determinants. The expression of prominin-1 in stem cells originating from various tissues suggests that it might be implicated in the determination of cell fate, although this remains to be elucidated [Kosodo et al., 2004]. Nevertheless, its dual association with plasma membrane protrusions and membrane microdomains deserves particular consideration. In the same way, the subcellular localization of prominin-1 might also be instructive with regard to the general organization of the plasma membrane of nonmigrating HSPCs. Thus, we have demonstrated that small microvillar-like structures, where prominin-1 is selectively concentrated [Corbeil et al., 2000], were randomly distributed over the entire surface of HSPCs either derived from peripheral blood immediately upon granulocyte colony-stimulating factor mobilization (fig. 2a) [Freund et al., 2006b] or freshly isolated from umbilical cord blood [Giebel et al., 2004]. A comparable distribution was observed for CD34 (fig. 2b), although this marker was not selectively associated with plasma membrane protrusions [Corbeil et al., 2000]. Interestingly, a similar analysis of hematopoietic cells derived from leukemic blasts revealed that, while CD34 was randomly distributed over the entire cell surface (fig. 2d), prominin-1 was concentrated on one side or pole, leading to the polarization of the plasma membrane of these cells (fig. $2 c$, solid line). Remarkably, the polarization of HSPCs also occurred upon contact with the feeder cell layer leading to the formation of a microvillar pole evidenced by the clustering of prominin-1 (fig. 2e), which could also be visualized by scanning electron microscopy (fig. 2f) [Freund et al., 2006b]. The physiological relevance of the microvillar pole is currently unknown, but the polarization of prominin-1 clearly reflects the plasma membrane compartmentalization of HSPCs. Such a phenomenon might be related to the quiescence or proliferation of HSPCs. In this particular context it is highly interesting to note that the hematopoietic cells including the CD133+ cell population are in $\mathrm{G}_{0} / \mathrm{G}_{1}$ phase immediately upon immunoisolation from umbilical cord blood [Grskovic et al., 2004], suggesting that the absence of a microvillar pole might be

Cells Tissues Organs 2008;188:127-138 
Leukapheresis product
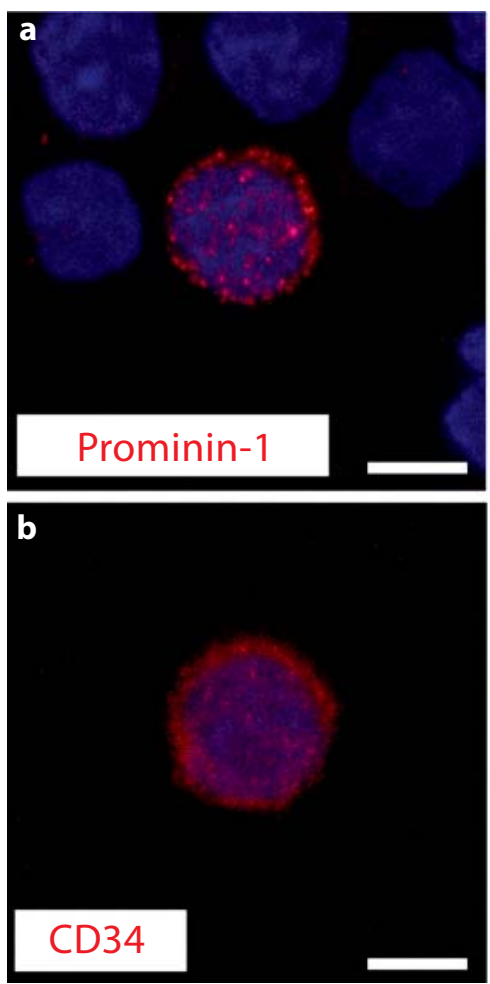

Leukemic blasts
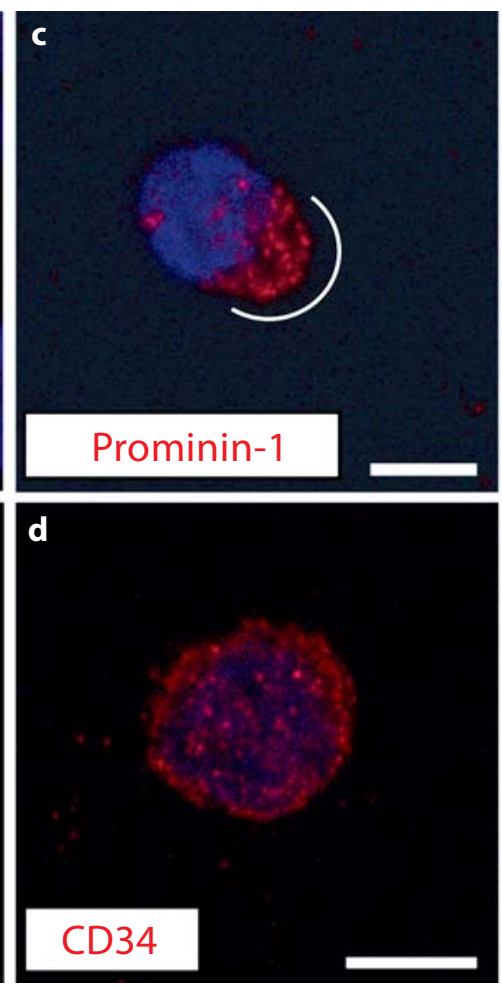

CD34+ isolated HSPCs growing on MSCs
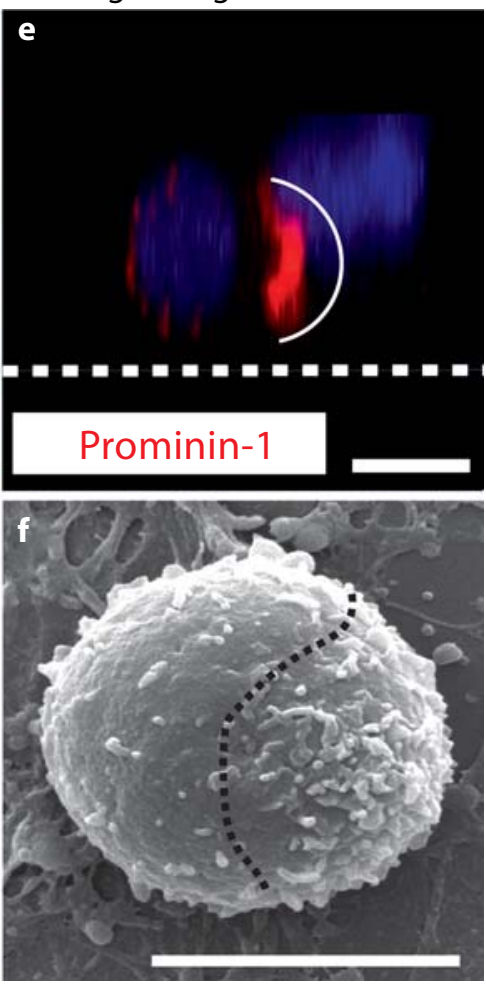

Fig. 2. Polarization of HSPCs and their leukemic counterparts as revealed by the subcellular localization of prominin-1. Human hematopoietic cells from leukapheresis products $(\mathbf{a}, \mathbf{b})$ and from an acute myeloid leukemia (c, d; subtype M2 according to FrenchAmerican-British classification) were fixed with $4 \%$ paraformaldehyde at room temperature prior to labeling with either mouse monoclonal antibody CD133/1 (anti-prominin-1; Miltenyi Biotec) or mouse monoclonal antibody CD34 (clone 581; BD Pharmingen) followed by Cy3-conjugated goat anti-mouse IgG (Jackson Immunoresearch). Nuclei were visualized with 4,6-diamidino-2phenylindrole. The immunofluorescence analysis was performed using a confocal microscope. $\mathbf{a}-\mathbf{d}$ A composite $\mathrm{x}-\mathrm{y}$ picture from 8 optical sections is shown. e, f In the coculture experiment, CD34+ MACS-immunoisolated cells (HSPCs) from leukapheresis products were cultured for 7 days on primary human MSCs prior to the labeling procedure as described previously [Freund et al., 2006b]. e A single $\mathrm{z}$ section is shown. Solid lines indicate the polarization of prominin-1; the dashed line shows the level of MSC. f Scanning electron microscopy analysis of HSPCs growing on MSCs reveals the presence of a microvillar pole (dotted line) on HSPCs exhibiting a round morphology. Scale bars $=5 \mu \mathrm{m}$. correlated to a quiescent state of the cells. Vice versa, HSPC polarization might be related to proliferation, which was exactly the case when the HSPCs were cultured ex vivo [Freund et al., 2006b]. The same holds true for their leukemic counterparts. Thus, polarization of HSPCs might be an indicator of their proliferative status. Further studies involving time lapse video microscopy are needed to dissect this issue.

A detailed analysis of various phases of mitotic HSPCs, derived from leukapheresis products, growing on MSCs as a feeder cell layer, revealed a complex distribution of prominin-1 (for details, see fig. 3 in Fargeas et al. [2006]). For instance, prominin-1, but not CD34, becomes con- centrated in surface domains corresponding to the spindle pole region during metaphase. In early and late anaphase, prominin-1 is enriched towards the cleavage furrow, and in some cases, also remained clustered at 1 pole of the dividing cells [Fargeas et al., 2006; Beckmann et al., 2007]. This concentration of prominin-1 in the cleavage furrow is consistent with the enrichment of microvillarlike structures therein, as observed in the RBL-2H3 cell line [Yonemura et al., 1993]. In telophase and cytokinesis, prominin-1 is either equally or unequally distributed between the 2 nascent daughter cells (fig. 3, left) [Fargeas et al., 2006]. A similar symmetric or asymmetric distribution of prominin-1 was observed when the HSPCs were 
Fig. 3. Symmetric and asymmetric cell division of HSPCs growing on various surfaces. CD34+ MACS-immunoisolated cells (HSPCs) from leukapheresis products were cultured for 3 days on either primary human MSCs, or fibronectin-coated or uncoated glass coverslips prior to the labeling procedure [Fargeas et al., 2006]. Paraformaldehyde-fixed, saponin-permeabilized cells were labeled with mouse monoclonal antibody CD133/1 (antiprominin-1; Miltenyi Biotec; red) and rat monoclonal antibody anti- $\alpha$-tubulin (Serotec; green) followed by appropriate Cy3and Cy2-conjugated secondary antibodies before double immunofluorescence analysis using confocal microscopy. Nuclei were visualized with 4,6-diamidino-2-phenylindrole. A composite of 9 optical sections is shown. White arrows indicate the midbody. Scale bars $=5 \mu \mathrm{m}$.
HSPCs growing on MSCs
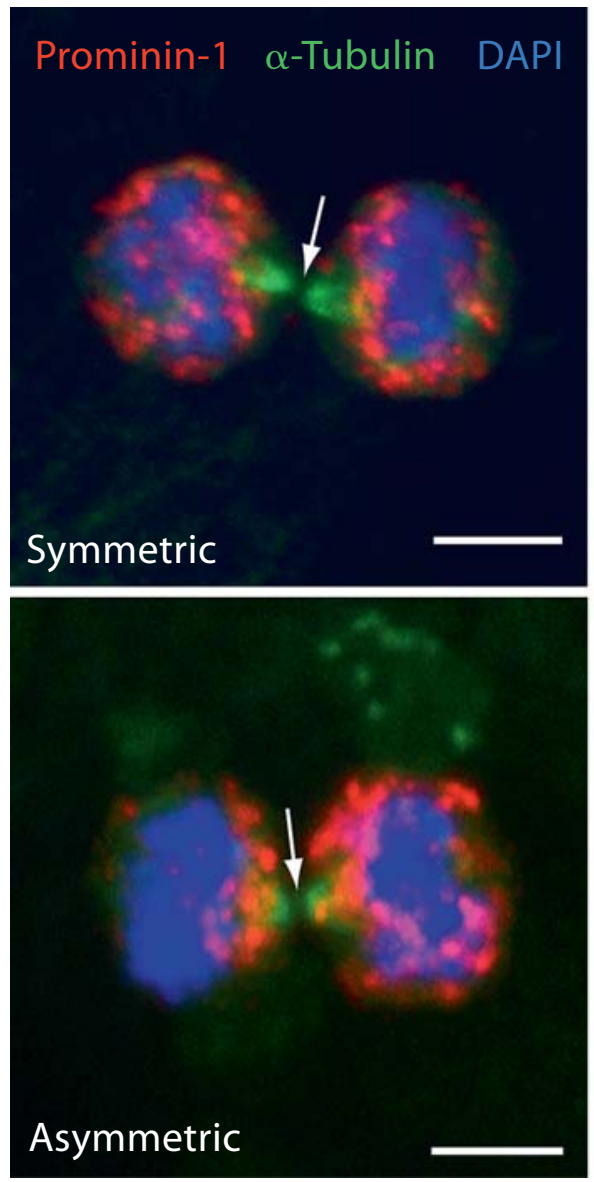

HSPCs growing on fibronectin

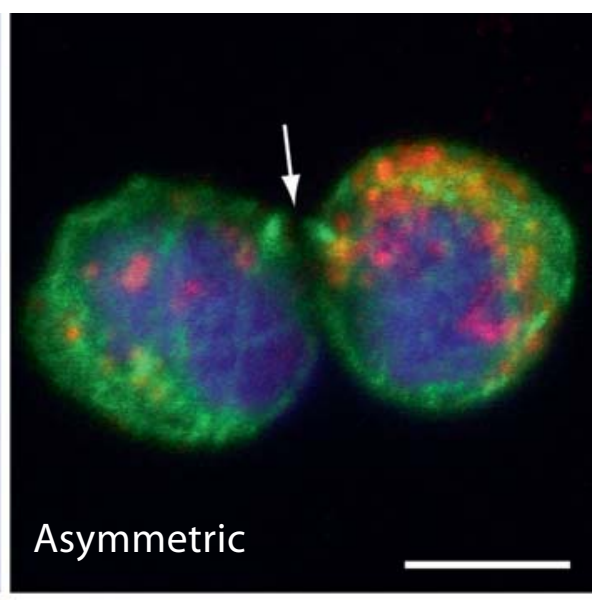

HSPCs growing on glass

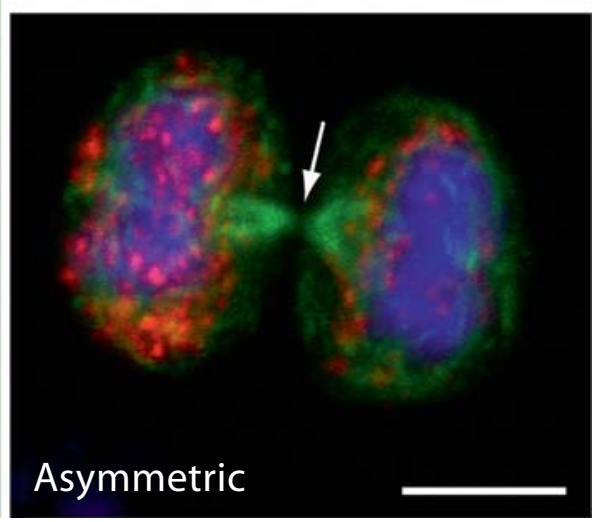

cultured either on fibronectin-coated or uncoated glass coverslips, indicating that such a phenomenon is independent of extrinsic factors (fig. 3, right; data not shown). These observations are not unique to the hematopoietic cells, since a similar phenomenon, that is, symmetric versus asymmetric distribution of prominin-1, has already been described in mammalian neuroepithelial progenitors [Kosodo et al., 2004]. During neurogenesis, prominin-1 is also highly concentrated in midbodies of symmetrically dividing neuroepithelial progenitors [Dubreuil et al., 2007], in contrast to HSPCs (fig. 3, arrows) [Fargeas et al., 2006]. However, some investigators have recently observed a certain amount of prominin-1 in midbodies of dividing HSPCs derived from umbilical cord blood [Beckmann et al., 2007]. Future studies are necessary to determine the cell biological consequences of such an asymmetric distribution of prominin-1, but knowing that this cholesterol-binding protein is associated with a specific membrane microdomain (see above), one might consider that its unequal distribution reflects the asymmetric distribution of these microdomains. The concept of stem cell-specific membrane microdomains carrying all molecular determinants necessary to maintain the stem cell properties is highly appealing in this context. Alternatively, these stem cell membrane microdomains might also be eliminated from the plasma membrane either by selective release from microvillar-like structures as membrane vesicles, such as the prominosomes described above [Marzesco et al., 2005; Bauer et al., 2007, manuscript in preparation], or, in HSPCs, by endocytosis into the multivesicular bodies and eventual release with exosomes [Bauer et al., 2007]. Both mechanisms are not mutually exclusive, although the association of prominin-1 with multivesicular bodies has not been reported in neuroepithelial cells [Marzesco et al., 2005; Dubreuil et al., 2007]. 


\section{Conclusions}

In conclusion, prominin- 1 , as a stem and progenitor marker, has rapidly become a tool generally used to define a self-renewing cell population in various tissues, which is highly important for tissue engineering and stem cell-based therapies. Its association with potential cancer stem cells might contribute to outline new prospects for more effective cancer therapy by targeting tumor-initiating cells. As a marker of plasma membrane protrusions and membrane microdomains, prominin-1 has already highlighted novel cell biological aspects, particularly regarding the general organization of the plasma membrane of stem and progenitor cells. In the future, the prominin-1-containing membrane microdomain associated with these primitive cells and the prominosomes released by them should reveal the peculiar mechanisms underlying stem cell properties.

\section{Acknowledgments}

The authors acknowledge Professor Wieland B. Huttner for helpful discussion. M.B. was supported by a grant from the Deutsche Forschungsgemeinschaft (DFG; SFB 655 A12) and D.C. was supported by grants from the DFG (SPP 1109, CO 298/2-2; SFB/TR13-04 B1; SFB 655 A13), the Center for Regenerative Therapies Dresden and the Sächsisches Ministerium für Wissenschaft und Kunst - Europäischer Fond für Regionale Entwicklung (4212/05-16).

\section{References}

Adams, G.B., D.T. Scadden (2006) The hematopoietic stem cell in its place. Nat Immunol 7: 333-337.

Bauer, N., M. Wilsch-Bräuninger, A.V. Fonseca, D. Freund, T. Baust, M. Bornhäuser, B. Hoflack, W.B. Huttner, D. Corbeil (2007) The stem cell marker prominin-1 (CD133) is released from hematopoietic stem and progenitor cells in association with small membrane vesicles (abstract 1505). International Society for Stem Cell Research (ISSCR) Meeting, Cairns.

Beaujean, F. (1997) Methods of CD34+ cell separation: comparative analysis. Transfus Sci 18: 251-261.

Beckmann, J., S. Scheitza, P. Wernet, J.C. Fischer, B. Giebel (2007) Asymmetric cell division within the human hematopoietic stem and progenitor cell compartment: Identification of asymmetrically segregating proteins. Blood 109: 5494-5501.

Bhatia, M. (2001) AC133 expression in human stem cells. Leukemia 15: 1685-1688.

Bitan, M., M. Shapira, I. Resnick, I. Zilberman, S. Miron, S. Samuel, A. Ackerstein, S. Elad, S. Israel, A. Amar, E. Fibach, R. Or, S. Slavin (2005) Successful transplantation of haploidentically mismatched peripheral blood stem cells using $\mathrm{CD}_{133^{+}}$-purified stem cells Exp Hematol 33: 713-718.

Bonnet, D., J.D. Dick (1997) Human acute myeloid leukemia is organized as a hierarchy that originates from a primitive hematopoietic cell. Nature Med 3: 730-737.

Bornhäuser, M., L. Eger, U. Oelschlaegel, S. Auffermann-Gretzinger, A. Kiani, J. Schetelig, T. Illmer, M. Schaich, D. Corbeil, C. Thiede, G. Ehninger (2005) Rapid reconstitution of dendritic cells after allogeneic transplantation of CD133+ selected hematopoietic stem cells. Leukemia 19: 161-165.
Brenner, S., M.F. Ryser, N.L. Whiting-Theobald, M. Gentsch, G.F. Linton, H.L. Malech (2007) The late dividing population of gamma-retroviral vector transduced human mobilized peripheral blood progenitor cells contributes most to gene-marked cell engraftment in NOD/SCID mice. Stem Cells 25: 1807-1813.

Butcher, E.C., L.J. Picker (1996) Lymphocyte homing and homeostasis. Science 272: 60 66.

Cancelas, J.A., M. Jansen, D.A. Williams (2006) The role of chemokine activation of Rac GTPases in hematopoietic stem cell marrow homing, retention, and peripheral mobilization. Exp Hematol 34: 976-985.

Corbeil, D., K. Röper, M.J. Hannah, A. Hellwig, W.B. Huttner (1999) Selective localization of the polytopic membrane protein prominin in microvilli of epithelial cells - a combination of apical sorting and retention in plasma membrane protrusions. J Cell Sci 112: 10231033.

Corbeil, D., K. Röper, A. Hellwig, M. Tavian, S. Miraglia, S.M. Watt, P.J. Simmons, B. Peault, D.W. Buck, W.B. Huttner (2000) The human AC133 hematopoietic stem cell antigen is also expressed in epithelial cells and targeted to plasma membrane protrusions. J Biol Chem 275: 5512-5520.

Corbeil, D., K. Röper, C.A. Fargeas, A. Joester, W.B. Huttner (2001) Prominin: A story of cholesterol, plasma membrane protrusions and human pathology. Traffic 2: 82-91.

Dar, A., O. Kollet, T. Lapidot (2006) Mutual, reciprocal SDF-1/CXCR4 interactions between hematopoietic and bone marrow stromal cells regulate human stem cell migration and development in NOD/SCID chimeric mice. Exp Hematol 34: 967-975. de Wynter, E.A., D. Buck, C. Hart, R. Heywood, L.H. Coutinho, A. Clayton, J.A. Rafferty, D. Burt, G. Guenechea, J.A. Bueren, D. Gagen, L.J. Fairbairn, B.I. Lord, N.G. Testa (1998) CD34+AC133+ cells isolated from cord blood are highly enriched in long-term culture-initiating cells, NOD/SCID-repopulating cells and dendritic cell progenitors. Stem Cells 16: 387-396.

Dexter, T.M., T.D. Allen, L.G. Lajtha (1977) Conditions controlling the proliferation of haemopoietic stem cells in vitro. J Cell Physiol 91: 335-344.

Dubreuil, V., A.M. Marzesco, D. Corbeil, W.B. Huttner, M. Wilsch-Bräuninger (2007) Midbody and primary cilium of neural progenitors release extracellular membrane particles enriched in the stem cell marker prominin-1. J Cell Biol 176: 483-495.

Faber, A., C. Roderburg, F. Wein, R. Saffrich, A. Seckinger, K. Horsch, A. Diehlmann, D. Wong, G. Bridger, V. Eckstein, A.D. Ho, W. Wagner (2007) The many facets of SDF- $1 \alpha$, CXCR4 agonists and antagonists on hematopoietic progenitor cells. J Biomed Biotechnol DOI: $10.1155 / 2007 / 26065$

Fargeas, C.A., D. Corbeil, W.B. Huttner (2003) AC133 antigen, CD133, prominin-1, prominin-2, etc.: prominin family gene products in need of a rational nomenclature. Stem Cells 21: 506-508.

Fargeas, C.A., A. Joester, E. Missol-Kolka, A. Hellwig, W.B. Huttner, D. Corbeil (2004) Identification of novel Prominin-1/CD133 splice variants with alternative $\mathrm{C}$-termini and their expression in epididymis and testis. J Cell Sci 117: 4301-4311.

Fargeas, C.A., A.V. Fonseca, W.B. Huttner, D. Corbeil (2006) Prominin-1 (CD133): from progenitor cells to human diseases. Future Lipidol 1: 213-225. 
Fargeas, C.A., W.B. Huttner, D. Corbeil (2007) Nomenclature of prominin-1 (CD133) splice variants - an update. Tiss Antig 69: 602606.

Février, B., G. Raposo (2004) Exosomes: endosomal-derived vesicles shipping extracellular messages. Curr Opin Cell Biol 16: 415421.

Florek, M., M. Haase, A.M. Marzesco, D. Freund, G. Ehninger, W.B. Huttner, D. Corbeil (2005) Prominin-1/CD133, a neural and hematopoietic stem cell marker, is expressed in adult human differentiated cells and certain types of kidney cancer. Cell Tiss Res 319: 15-26.

Florek, M., N. Bauer, P. Janich, M. Wilsch-Bräuninger, C.A. Fargeas, A.M. Marzesco, G. Ehninger, C. Thiede, W.B. Huttner, D. Corbeil (2007) Prominin-2 is a cholesterolbinding protein associated with apical and basolateral plasmalemmal protrusions in polarized epithelial cells and released into the urine. Cell Tiss Res 328: 31-47.

Freund, D., J. Oswald, S. Feldmann, G. Ehninger, D. Corbeil, M. Bornhäuser (2006a) Comparative analysis of proliferative potential and clonogenicity of MACS-immunomagnetic isolated CD34+ and CD133+ blood stem cells derived from a single donor. Cell Prolif 39: 325-332.

Freund, D., N. Bauer, S. Boxberger, S. Feldmann, U. Streller, G. Ehninger, C. Werner, M. Bornhäuser, J. Oswald, D. Corbeil (2006b) Polarization of human hematopoietic progenitors during contact with multipotent mesenchymal stromal cells - effects on proliferation and clonogenicity. Stem Cells Dev 15: 815829.

Frimberger, A.E., C.I. McAuliffe, K.A. Werme, R.A. Tuft, K.E. Fogarty, B.O. Benoit, M.S Dooner, P.J. Quesenberry (2001) The fleet feet of haematopoietic stem cells: rapid motility, interaction and proteopodia. $\mathrm{Br} \mathrm{J} \mathrm{Hae-}$ matol 112: 644-654.

Fruehauf, S., K. Srbic, R. Seggewiss, J. Topaly, A.D. Ho (2002) Functional characterization of podia formation in normal and malignant hematopoietic cells. J Leukoc Biol 71: 425432.

Gallacher, L., B. Murdoch, D.M. Wu, F.N. Karanu, M. Keeney, M. Bhatia (2000) Isolation and characterization of human CD34- $\mathrm{Lin}^{-}$ and $\mathrm{CD} 34^{+} \mathrm{Lin}^{-}$hematopoietic stem cells using cell surface markers AC133 and CD7. Blood 95: 2813-2820.

Gehling, U.M., S. Ergun, U. Schumacher, C. Wagener, K. Pantel, M. Otte, G. Schuch, P. Schafhausen, T. Mende, N. Kilic, K. Kluge, B. Schafer, D.K. Hossfeld, W. Fiedler (2000) In vitro differentiation of endothelial cells from AC133-positive progenitor cells. Blood 95: 3106-3112.

Gerdes, H.H., N.V. Bukoreshtliev, J.F.V. Barroso (2007) Tunneling nanotubes: a new route for the exchange of components between animal cells. FEBS Lett 581: 2194-2201.
Ghiaur, G., A. Lee, J. Bailey, J.A. Cancelas, Y. Zheng, D.A. Williams (2006) Inhibition of RhoA GTPase activity enhances hematopoietic stem and progenitor cell proliferation and engraftment. Blood 108: 2087-2094.

Giebel, B., D. Corbeil, J. Beckmann, J. Höhn, D. Freund, K. Giesen, J. Fischer, G. Kögler, P. Wernet (2004) Segregation of lipid raft markers including CD133 in polarized human hematopoietic stem and progenitor cells. Blood 104: 2332-2338.

Gillette, J.M., F. Pruefer, J. Zhou, J. LippincottSchwartz (2006) Live cell imaging of interactions between hematopoietic progenitor cells and osteoblastic cells (abstract 2106). American Society for Cell Biology Meeting, San Diego.

Gómez-Moutón, C., J. Abad, E. Mira, R. Lacalle, E. Gallardo, S. Jíménez-Baranda, I. Illa, A. Bernad, S. Mañes, C. Martínez-A (2001) Segregation of leading-edge and uropod components into specific lipid rafts during $\mathrm{T}$ cell polarization. Proc Natl Acad Sci USA 98: 9642-9647.

Gordon, P.R., T. Leimig, A. Babarin-Dorner, J. Houston, M. Holladay, I. Mueller, T. Geiger, R. Handgretinger (2003) Large-scale isolation of CD133+ progenitor cells from G-CSF mobilized peripheral blood stem cells. Bone Marrow Transpl 31: 17-22.

Grskovic, B., K. Ruzicka, A. Karimi, D. Qujeq, M.M. Müller (2004) Cell cycle analysis of the CD133+ and CD133 - cells isolated from umbilical cord blood. Clin Chim Acta 343: $173-$ 178.

Hardy, C.L. (1995) The homing of hematopoietic stem cells to the bone marrow. Am J Med Sci 309: 260-266.

Janich, P., D. Corbeil (2007) GM1 and GM3 gangliosides highlight distinct lipid microdomains within the apical domain of epithelial cells. FEBS Lett 581: 1783-1787.

Jászai, J., C.A. Fargeas, M. Florek, W.B. Huttner, D. Corbeil (2007a) Focus on Molecules: Prominin-1 (CD133). Exp Eye Res 85: 585586.

Jászai, J., P. Janich, L.M. Farkas, C.A. Fargeas, W.B. Huttner, D. Corbeil (2007b) Differential expression of prominin-1 (CD133) and prominin-2 in major cephalic exocrine glands of adult mice. Histochem Cell Biol 128: 409-419.

Kadereit, S., L.S. Deeds, S.E. Haynesworth, O.N Koc, M.M. Kozik, E. Szekely, K. DaumWoods, G.W. Goetchius, P. Fu, L.A. Welniak, W.J. Murphy, M.J. Laughlin (2002) Expansion of LTC-ICs and maintenance of p21 and BCL-2 expression in cord blood $\mathrm{CD}_{34}{ }^{+} /$ $\mathrm{CD} 38^{-}$early progenitors cultured over human MSCs as a feeder layer. Stem Cells 20: 573-582.

Kania, G., D. Corbeil, J. Fuchs, K.V. Tarasov, P. Blyszczuk, W.B. Huttner, K.R. Boheler, A.M. Wobus (2005) The somatic stem cell marker prominin-1/CD133 is expressed in embryonic stem cell-derived progenitors. Stem Cells 23: 791-804.
Kiel, M.J., S.J. Morrison (2006) Maintaining hematopoietic stem cells in the vascular niche. Immunity 25: 862-864.

Koehl, U., S. Zimmermann, R. Esser, J. Sorensen, H.P. Gruttner, M. Duchscherer, E. Seifried, T. Klingebiel, D. Schwabe (2002) Autologous transplantation of CD133 selected hematopoietic progenitor cells in a pediatric patient with relapsed leukemia. Bone Marrow Transplant 29: 927-930.

Konakahara, S., K. Ohashi, K. Mizuno, K. Itoh, T. Tsuji (2004) CD29 integrin- and LIMK1/ cofilin-mediated actin reorganization regulates the migration of haematopoietic progenitor cells underneath bone marrow stromal cells. Genes Cells 9: 354-358.

Kosodo, Y., K. Röper, W. Haubensak, A.-M. Marzesco, D. Corbeil, W.B. Huttner (2004) Asymmetric distribution of the apical plasma membrane during neurogenic divisions of mammalian neuroepithelial cells. EMBO J 23: 2314-2324.

Kratz-Albers, K., M. Zuhlsdorp, R. Leo, W.L. Berdel, T. Buchner, H. Serve (1998) Expression of a AC133, a novel stem cell marker, on human leukemic blasts lacking CD34-antigen and on a human CD34+ leukemic line: MUTZ-2. Blood 92: 4485-4487.

Krause, D.S., M.J. Fackler, C.I. Civin, W.S. May (1996) CD34: structure, biology, and clinical utility. Blood 87: 1-13.

Kuci, S., J.T. Wessels, H.J. Buhring, K. Schilbach, M. Schumm, G. Seitz, J. Loffler, P. Bader, P.G. Schlegel, D. Niethammer, R. Handgretinger (2003) Identification of a novel class of human adherent CD34- stem cells that give rise to SCID-repopulating cells. Blood 101: 869-876.

Lang, P., P. Bader, M. Schumm, T. Feuchtinger, H. Einsele, M. Fuhrer, C. Weinstock, R. Handgretinger, S. Kuci, D. Martin, D. Niethammer, J. Greil (2004) Transplantation of a combination of CD133+ and CD34+ selected progenitor cells from alternative donors. $\mathrm{Br}$ J Haematol 124: 72-79.

Lapidot, T., O. Kollet (2002) The essential roles of the chemokine SDF-1 and its receptor CXCR4 in human stem cell homing and repopulation of transplanted immune-deficient NOD/SCID and NOD/SCID/B2m ${ }^{\text {null }}$ mice. Leukemia 16: 1992-2003.

Lardon, J., D. Corbeil, W.B. Huttner, Z. Ling, L. Bouwens (2007) Stem cell marker prominin 1/AC133 is expressed in duct cells of the adult human pancreas. Pancreas, in press.

Lee, A., J.D. Kessler, T.A. Read, C. Kaiser, D. Corbeil, W.B. Huttner, J.E. Johnson, R.J. Wechsler-Reya (2005) Isolation of neural stem cells from postnatal cerebellum. Nat Neurosci 8: 723-729.

Li, Z., L. Li (2006) Understanding hematopoietic stem-cell microenvironments. Trends Biochem Sci 31: 589-595.

Luster, A.D., R. Alon, U.H. von Adrian (2005) Immune cell migration in inflammation: present and future therapeutic targets. Nat Immunol 6: 1182-1190. 
Majumdar M.K., M.A. Thiede, S.E. Haynesworth, S.P. Bruder, S.L. Gerson (2000) Human marrow-derived mesenchymal stem cells (MSCs) express hematopoietic cytokines and support long-term hematopoiesis when differentiated toward stromal and osteogenic lineages. J Hematother Stem Cell Res 9: 841-848.

Marzesco, A.-M., P. Janich, M. Wilsch-Bräuninger, V. Dubreuil, K. Langenfeld, D. Corbeil, W.B. Huttner (2005) Release of extracellular membrane particles carrying the stem cell marker prominin-1 (CD133) from neural progenitors and other epithelial cells. J Cell Sci 118: 2849-2858.

Matsumoto, K., K. Yasui, N. Yamashita, Y. Horie, T. Yamada, Y. Tani, H. Shibata, T. Nakano (2000) In vitro proliferation potential of AC133 positive cells in peripheral blood. Stem Cells 18: 196-203.

Maw, M.A., D. Corbeil, J. Koch, A. Hellwig, J.C. Wilson-Wheeler, R.J. Bridges, G. Kumaramanickavel, S. John, D. Nancarrow, K. Röper, A. Weigmann, W.B. Huttner, M.J. Denton (2000) A frameshift mutation in prominin (mouse)-like 1 causes human retinal degeneration. Hum Mol Genet 9: 27-34.

Miraglia, S., W. Godfrey, A.H. Yin, K. Atkins, R. Warnke, J.T. Holden, R.A. Bray, E.K. Waller, D.W. Buck (1997) A novel five-transmembrane hematopoietic stem cell antigen: isolation, characterization, and molecular cloning. Blood 90: 5013-5021.

Nguyen, D.H., J.E. Hildreth (2000) Evidence for budding of human immunodeficiency virus type 1 selectively from glycolipid-enriched membrane lipid rafts. J Virol 74: 32643272.

O'Brien, C.A., A. Pollett, S. Gallinger, J.E. Dick (2007) A human colon cancer cell capable of initiating tumour growth in immunodeficient mice. Nature 445: 106-110.

Padovan, C.S., K. Jahn, T. Birnbaum, P. Reich, P. Sostak, M. Strupp, A. Straube (2003) Expression of neuronal markers in differentiated marrow stromal cells and CD133+ stem-like cells. Cell Transpl 12: 839-848.

Papayannopoulou, T., C. Craddock, B. Nakamoto, G.V. Priestly, N.S. Wolf (1995) The VLA4/ VCAM-1 adhesion pathway defines contrasting mechanisms of lodgement of transplanted murine hematopoietic progenitors between bone marrow and spleen. Proc Natl Acad Sci USA 92: 9647-9651.

Pike, L.J. (2004) Lipid rafts: heterogeneity on the high seas. Biochem J 378: 281-292.

Pike, L.J. (2006) Rafts defined: a report on the Keystone Symposium on Lipid Rafts and Cell Function. J Lipid Res 47: 1597-1598.
Quirici, N., D. Soligo, L. Caneva, F. Servida, P. Bossolasco, G.L. Deliliers (2001) Differentiation and expansion of endothelial cells from human bone marrow $\mathrm{CD}_{133^{+}}$cells. Br J Haematol 115: 186-194.

Ricci-Vitiani, L., D.G. Lombardi, E. Pilozzi, M. Biffoni, M. Todaro, C. Peschle, R. De Maria (2007) Identification and expansion of human colon-cancer-initiating cells. Nature 445: 111-115.

Richardson, G.D., C.N. Robson, S.H. Lang, D.E. Neal, N.J. Maitland, A.T. Collins (2004) CD133, a novel marker for human prostatic epithelial stem cells. J Cell Sci 117: 35393545.

Röper, K., D. Corbeil, W.B. Huttner (2000) Retention of prominin in microvilli reveals distinct cholesterol-based lipid micro-domains in the apical plasma membrane. Nat Cell Biol 2: 582-592.

Rustom, A., R. Saffrich, I. Markovic, P. Walther, H.H. Gerdes (2004) Nanotubular highways for intercellular organelle transport. Science 303: $1007-1010$.

Sanchez-Madrid, F., M.A. del Pozo (1999) Leukocyte polarization in cell migration and immune interactions. EMBO J 18: 501-511.

Simons, K., D. Toomre (2000) Lipid rafts and signal transduction. Nat Rev Mol Cell Biol 1: 31-39.

Singh, S.K., C. Hawkins, I.D. Clarke, J.A. Squire, J. Bayani, T. Hide, R.M. Henkelman, M.D. Cusimano, P.B. Dirks (2004) Identification of human brain tumour initiating cells. $\mathrm{Na}$ ture 432: 396-401.

Torrente, Y., M. Belicchi, M. Sampaolesi, F. Pisati, M. Meregalli, G. D'Antona, R. Tonlorenzi, L. Porretti, M. Gavina, K. Mamchaoui, M.A. Pellegrino, D. Furling, V. Mouly, G.S. Butler-Browne, R. Bottinelli, G. Cossu, N. Bresolin (2004) Human circulating $\mathrm{AC} 133^{+}$stem cells restore dystrophin expression and ameliorate function in dystrophic skeletal muscle. J Clin Invest 114: 182-195.

Uchida, N., D.W. Buck, D. He, M.J. Reitsma, M. Masek, T.V. Phan, A.S. Tsukamoto, F.H. Gage, I.L. Weissman (2000) Direct isolation of human central nervous system stem cells. Proc Natl Acad Sci USA 97: 14720-14725.

Van Overstraeten-Schlogel, N., Y. Beguin, A. Gothot (2006) Role of stromal-derived factor-1 in the hematopoietic-supporting activity of human mesenchymal stem cells. Eur J Haematol 76: 488-493.

Varnum-Finney, B., L. Wu, M. Yu, C. BrashemStein, S. Staats, D. Flowers, J.D. Griffin, I.D. Bernstein (2000) Immobilization of Notch ligand, Delta-1, is required for induction of notch signaling. J Cell Sci 113: 4313-4318.
Wagner, W., R. Saffrich, U. Wirkner, V. Eckstein, J. Blake, A. Ansorge, C. Schwager, F. Wein, K. Miesala, W. Ansorge, A.D. Ho (2005) Hematopoietic progenitor cells and cellular microenvironment: behavioral and molecular changes upon interaction. Stem Cells 23: 1180-1191.

Wagner, W., F. Wein, C. Roderburg, R. Saffrich, A. Faber, U. Krause, M. Schubert, V. Benes, V. Eckstein, H. Maul, A.D. Ho (2007) Adhesion of hematopoietic progenitor cells to human mesenchymal stem cells as a model for cell-cell interaction. Exp Hematol 35: $314-$ 325.

Weigmann, A., D. Corbeil, A. Hellwig, W.B. Huttner (1997) Prominin, a novel microvillispecific polytopic membrane protein of the apical surface of epithelial cells, is targeted to plasmalemmal protrusions of non-epithelial cells. Proc Natl Acad Sci USA 94: 1242512430.

Wilson, A., A. Trumpp (2006) Bone-marrow haematopoietic-stem-cell niches. Nat Rev Immunol 6: 93-106.

Wright, D.E., A.J. Wagers, A.P. Gulati, F.L. Johnson, I.L. Weissman (2001) Physiological migration of hematopoietic stem and progenitor cells. Science 294: 1933-1936.

Yang, L., L. Wang, H. Geiger, J.A. Cancelas, J. Mo, Y. Zheng (2007) Rho GTPase Cdc42 coordinates hematopoietic stem cell quiescence and niche interaction in the bone marrow. Proc Natl Acad Sci USA 104: $5091-$ 5096.

Yin, A.H., S. Miraglia, E.D. Zanjani, G. AlmeidaPorada, M. Ogawa, A.G. Leary, J. Olweus, J. Kearney, D.W. Buck (1997) AC133, a novel marker for human hematopoietic stem and progenitor cells. Blood 90: 5002-5012.

Yonemura, S., A. Nagafuchi, N. Sato, S. Tsukita (1993) Concentration of an integral membrane protein, CD43 (leukosialin, sialophorin), in the cleavage furrow through the interaction of its cytoplasmic domain with actin-based cytoskeletons. J Cell Biol 120 : 437-449.

Yu, Y., A. Flint, E.L. Dvorin, J. Bischoff (2002) AC133-2, a novel isoform of human AC133 stem cell antigen. J Biol Chem 277: 2071120716.

Zhang, Q., F. Zulfiqar, X. Xiao, S.A. Riazuddin. Z. Ahmad, R. Caruso, I. Macdonald, P. Sieving, S. Riazuddin, J.F. Hejtmancik (2007) Severe retinitis pigmentosa mapped to $4 \mathrm{p} 15$ and associated with a novel mutation in the PROM1 gene. Hum Genet 122: 293-299. 